\title{
ATUAÇÃO DOS PROFISSIONAIS DE ENFERMAGEM NA PREVENÇÃO E CONTROLE DAS INFECÇÕES RELACIONADAS À ASSISTÊNCIA À SAÚDE
}

\section{ACTION OF NURSING PROFESSIONALS IN THE PREVENTION AND CONTROL OF HEALTH CARE-INFECTED INFECTIONS}

\author{
Marcelo Flavio Batista da Silva \\ Jefferson da Silva Santana
}

Caio Clayderman Ferreira de Lima e Silva

\section{RESUMO}

As infecções relacionadas à assistência à saúde são um risco que ameaça a segurança do paciente, sendo causa de morbimortalidade, desafiando os avanços científico-tecnológicos e, mobilizando a atenção de profissionais, pesquisadores, organizações nacionais e internacionais que buscam a efetividade das medidas de prevenção e controle. O objetivo deste trabalho foi identificar o conhecimento produzido a respeito da temática. É uma revisão integrativa, que permite examinar estudos científicos de forma sistemática e abrangente. No contexto dos serviços de saúde, destaca-se como uma das principais preocupações em relação à segurança do paciente e qualidade dos serviços a redução da incidência das Infecções Relacionadas à Saúde. Assim, vigilância, prevenção e controle têm se tornados complexos nas últimas décadas, devido ao impacto dessas infecções sobre letalidade hospitalar, duração da internação e custos. Apoiado nesse entendimento, este estudo apresenta como inovação a consideração de que a Educação Permanente em Saúde constitui-se em estratégia que respalda o desenvolvimento de ações de Educação em Saúde e Educação em Serviço, desde que sejam operacionalizadas por metodologias que promovam o empoderamento e a qualificação profissional e nisso entra o papel de educador do enfermeiro.

Descritores: Controle de Doenças; Educação Permanente; Infecção Hospitalar; Prevenção de Doenças; Profissionais de Enfermagem.

\section{ABSTRACT}

Infections related to health care are a risk that threatens the patient's safety, causing morbidity and mortality, challenging scientific and technological advances and mobilizing the attention of professionals, researchers, national and international organizations that seek the effectiveness of prevention measures and control. The objective of this work was to identify the knowledge produced on the subject. It is an 
integrative review, which allows to examine scientific studies in a systematic and comprehensive way. In the context of health services, one of the main concerns regarding patient safety and quality of services is the reduction of the incidence of health-related infections. Thus, surveillance, prevention and control have become complex in the last decades, due to the impact of these infections on hospital lethality, duration of hospitalization and costs. Based on this understanding, this study presents as an innovation the consideration that Permanent Education in Health is a strategy that supports the development of Health Education and In-Service Education actions, provided they are operationalized by methodologies that promote empowerment and professional qualification and in this enters the role of educator of the nurse.

Keywords: Disease Control; Permanent Education; Hospital Infection; Prevention of diseases; Nursing Professionals.

\section{INTRODUÇÃO}

As infecções relacionadas à assistência à saúde (IRAS), ou Infecções Hospitalares $(\mathrm{IH})$ como ainda são conceituadas por alguns pesquisadores, são um risco que ameaça a segurança do paciente, sendo causa de morbimortalidade, desafiando os avanços científico-tecnológicos e, mobilizando a atenção de profissionais, pesquisadores, organizações nacionais e internacionais que buscam a efetividade das medidas de prevenção e controle1.

Os mais suscetíveis são as crianças; porque possuem uma instabilidade fisiológica, permitindo que os mesmos contraiam a infecção mais facilmente; os idosos, devido às alterações fisiológicas proporcionadas pela senilidade e, em muitos casos, por estarem submetidos a tratamentos oncológicos, e cirúrgicos ou em UTI, em que o uso de antimicrobianos potentes e de largo espectro é a regra e os procedimentos invasivos são rotineiros e por último; têm-se os imunocomprometidos. O principal patógeno responsável por causar infecções no sítio hospitalar e possuir uma elevada resistência a antimicrobianos é o Staphylococcus aureus ${ }^{2}$.

No nosso país, as IRAS encontram-se em torno de 6\%, triplicando o percentual de tolerância da Organização Mundial de Saúde (OMS), fato este que agrava a situação dos hospitais brasileiros. O paciente que evolui para uma infecção pode levar a um gasto de até três vezes maior do que o custo do paciente sem infecção hospitalar ${ }^{3}$. 
O controle de infecção hospitalar foi regulamentado pelo Ministério da Saúde, através da criação do Programa Nacional de Controle de Infecção Hospitalar (PNCIH) em 1982 e com a promulgação da Lei Federal 9.431 que obrigou todos os hospitais brasileiros a constituírem uma Comissão de Controle de Infecção Hospitalar $(\mathrm{CCIH})$, encarregada de executar tarefas importantíssimas como: detectar os casos de infecção hospitalar, elaborar normas de padronização, colaborar com o treinamento de todos os profissionais de saúde, realizar controle da prescrição de antibióticos e oferecer apoio técnico à administração hospitalar ${ }^{4}$.

Contudo, os profissionais de enfermagem também são os responsáveis por prevenir e controlar sistematicamente os processos de contaminação que possam causar danos à clientela durante assistência de sua profissão³.

O Ministério da Saúde afirma que todos os profissionais que trabalham em serviços de saúde, que mantém contato direto ou indireto com os usuários, que atuam na manipulação de medicamentos, alimentos e material estéril ou contaminado devem adotar em sua prática as recomendações básicas de higienização das mãos (HM). Apesar da importância da transmissão de infecções pelo contato das mãos ser admitida mundialmente e sua efetividade comprovada, o cumprimento das normas técnicas para sua prevenção é insatisfatória ${ }^{5}$.

Diante desse panorama é imprescindível que haja um processo de formação/educação permanente do trabalhador, tendo o conhecimento das normas e legislação reguladora da prevenção às infecções, o que exige construção e reconstrução constante do conhecimento ${ }^{6}$.

Diante dessa argumentação e baseado no pressuposto de que este estudo é de fundamental importância, uma vez que a IRAS/IH estão em evidência nos serviços de saúde, em especial, nos hospitais, resolveu-se, então, como objetivo desta pesquisa, identificar o conhecimento produzido a respeito da atuação dos profissionais de enfermagem na prevenção e controle das IRAS, através de artigos científicos disponíveis em base de dado.

A relevância deste estudo pretende contribuir significativamente em pesquisas da área da saúde, pois a atualidade desta temática desvela-se na crescente demanda de pessoas em cuidados de saúde, levando à reflexão da importância do 
aprimoramento das ações de prevenção e controle das IRAS/IH, por parte dos profissionais de enfermagem.

\section{METODOLOGIA}

Tratou-se de uma revisão integrativa, que permite examinar estudos científicos de forma sistemática e abrangente ${ }^{7}$. Foram realizadas buscas na Biblioteca Virtual em Saúde (BVS), LILACS (Literatura Latino-Americana em Ciências da Saúde) e SCIELO (Scientific Eletronic Library on line) com os seguintes descritores: Controle de Doenças; Educação Permanente; Infecção Hospitalar; Prevenção de Doenças; Profissionais de Enfermagem. Como critérios de inclusão, utilizaram-se artigos que abordassem a temática, de produção nacional, em língua portuguesa, completos, publicados nos últimos 10 anos, disponíveis de forma on-line e gratuitos. Como critérios de exclusão: artigos não disponíveis na íntegra. Após leitura criteriosa dos resumos de 220 artigos pesquisados, foram excluídos 120 artigos por não contemplarem ou contemplarem somente parte da temática em estudo, ou, ainda, estarem presentes em mais de uma base de dados, resultando em um total de 100 artigos a serem lidos na íntegra.

A análise se deu através de: pré-análise, exploração do material e tratamento dos resultados obtidos. Após leitura criteriosa e refinamento, ficaram 27 artigos e 02 dissertações de mestrado que foram selecionados porque respondiam ao objetivo da temática. Foi respeitada a Lei do Direito Autoral em todas as citações. Alguns pesquisadores ainda utilizam o termo "Infecção Hospitalar" e o mesmo foi preservado, devido o respeito à autoria. A apresentação dos resultados e discussão foi feita de forma descritiva, possibilitando ao leitor a avaliação da aplicabilidade desta pesquisa.

\section{RESULTADOS E DISCUSSÃO INFECÇÃO RELACIONADA À ASSISTÊNCIA À SAÚDE - IRA}

Com os estudos desenvolvidos e a atualização desta temática, mudanças foram inevitáveis, dentre essas se destaca a criação do termo "Infecção relacionada à Assistência à Saúde (IRAS)", em substituição ao termo "Infecção Hospitalar" (IH), o 
qual amplia o cenário de práticas em saúde e assim, possíveis locais para surgimento dessas intercorrências ${ }^{8}$.

No contexto dos serviços de saúde, destaca-se como uma das principais preocupações em relação à segurança do paciente e qualidade dos serviços a redução da incidência das IRAS, que se caracterizam como infecções adquiridas durante o processo de cuidado em um hospital ou outra unidade prestadora de assistência à saúde, e que não estavam presentes ou em incubação na admissão do paciente. Tais infecções podem se manifestar durante a internação ou após a alta hospitalar. Assim, vigilância, prevenção e controle têm se tornados complexos nas últimas décadas, devido ao impacto dessas infecções sobre letalidade hospitalar, duração da internação e custos ${ }^{9}$.

Infecção é a penetração, alojamento, multiplicação de um microrganismo em um organismo humano produzindo-lhes danos, reduzindo as capacidades de defesa do indivíduo e de uma particular interação de diversos fatores do agente, meio e hospedeiro causando doenças ou síndromes infecciosas ${ }^{10}$.

O alto risco de mortalidade relacionada às IRAS está intimamente associado a fatores como a realização de procedimentos invasivos, diagnósticos e terapêuticos, à gravidade da doença de base que acomete o paciente, ao sítio da infecção, à adequação da terapia e à sensibilidade dos microrganismos aos antimicrobianos ${ }^{11}$.

Soma-se a esse quadro o aumento da expectativa de vida, o aumento das condições que induzem à internação de indivíduos cada vez mais graves e imunocomprometidos, a mudança em perfis epidemiológicos com doenças novas e emergentes e o surgimento de patógenos multirresistentes ${ }^{9}$.

Em meados do século XVIII, os hospitais se constituíam em fonte inesgotável de doença e de morte para indivíduos ali acolhidos devido a sua superlotação, carência de recursos, sua arquitetura, forma de organização e a falta de conhecimentos sobre a transmissão de doenças infectocontagiosas. Não existia, no entanto, o conceito de infecção hospitalar e o desenvolvimento de práticas específicas para sua prevenção e controle ainda não faziam parte das atividades ali desenvolvidas ${ }^{10}$.

A maioria das infecções hospitalares, (70\%), tem sua origem na microbiota endógena, em consequência da própria doença ou pela agressão diagnóstica e 
terapêutica a que o paciente foi submetido, e o restante, na microbiota exógena, sendo seus agentes procedentes através do ar, da água, dos artigos hospitalares ou medicamentos. A via de transmissão pode ser direta, indireta ou por vetores ${ }^{12}$.

Os índices de infecção funcionam como um parâmetro de controle de qualidade do serviço prestado por um hospital. Elas ocasionam custos consideráveis aos sistemas de saúde de todo o mundo, por isso o uso inadequado de antimicrobiano, o aumento dos dias de internação e os consequentes custos com terapêutica antimicrobiana, além das questões pessoais, como alterações psicológicas dos pacientes, evidenciam a importância da prevenção e controle das infecções hospitalares ${ }^{13}$.

Os dados sobre $\mathrm{IH}$ no Brasil são escassos e pouco divulgados porque não são consolidados por muitos hospitais, o que dificulta o conhecimento da dimensão do problema em âmbito nacional ${ }^{14}$.

A epidemiologia estuda os fatores que determinam a ocorrência e a distribuição da doença na população. Ela relaciona o que está acontecendo na vida dos indivíduos e da sociedade, a ocorrência e a distribuição de doenças, lesões e outros problemas se saúde. Ela se preocupa com todos os fatores biológicos, sociais comportamentais, espirituais e psicológicos que podem aumentar a frequência da doença, ou oferecer oportunidades para a prevenção. Além disso, as IRAS são tidas como principal causa de iatrogênia da pessoa hospitalizada e submetida a intervenções curativas ${ }^{12}$. Estimase que cerca de $60 \%$ delas sejam associadas a algum dispositivo intravascular ${ }^{15}$.

As mais comuns são infecção do trato urinário, pneumonia, infecção do sítio cirúrgico e sepse, com percentual variando, respectivamente, de $40,8 \%$ a $42 \%, 11 \%$ a 32,9\%, $8 \%$ a 24\%, 5\% a 9,2\%. No hospital, a infecção do trato urinário (ITU) acomete $2 \%$ dos pacientes internados, sendo responsáveis por $35 \%$ a $45 \%$ de todas as infecções acometidas no hospital, sendo essa a causa mais comum de infecção nosocomial. Entre os pacientes que são hospitalizados, mais de $10 \%$ são expostos temporariamente à caterização vesical de demora, o fator isolado mais importante que predispõe esses pacientes à infecção ${ }^{12}$. 


\section{CCIH - COMISSÃO DE CONTROLE DE INFECÇÃO HOSPITALAR}

O Controle de Infecção Hospitalar $(\mathrm{ClH})$ é entendido como um conjunto de ações implementadas buscando a redução da incidência e a prevenção destas. Para efeitos legais, todo hospital deve contar com uma Comissão de Controle de Infecções Hospitalares $(\mathrm{CClH})$, a qual deve orientar-se pela Portaria 2616, de 16 de maio de 1998, do Ministério da Saúde que estabelece diretrizes para a prevenção e controle de infecções ${ }^{16}$.

A CCIH é composta por profissionais de nível superior e da área da saúde que se dividem em membros consultores e executores, que tem a função de planejar, elaborar, implementar e avaliar o Programa de Controle de Infecção Hospitalar, adequando-o às necessidades da instituição hospitalar. Os Membros Consultores das CCIH "são os responsáveis pelo estabelecimento das diretrizes para o Programa de Controle de Infecção Hospitalar", representando os serviços médico, de enfermagem, de farmácia, de microbiologia e administração. Os Membros Executores são os profissionais, comumente enfermeiro e médico, que representam o SCIH e, portanto, "são encarregados da execução das ações programadas de controle de infecção hospitalar"16.

As Comissões de Controle de Infecção Hospitalar (CCIH) atendem prontamente a essa finalidade, em conjunto com os profissionais da saúde, através da adoção de condutas e precauções padrões e implementação de estratégias de educação permanente a esses profissionais a fim de melhorar suas ações, a assistência e a sobrevida dos pacientes ${ }^{17}$.

As comissões de controle de infecção usam vários meios para prevenir as infecções hospitalares, dentre estes, a higienização das mãos é muito importante na redução da transmissão dos microorganismos que causam infecção. Muitos são as pesquisas científicas que demonstram essa redução, porém embora existam campanhas para o controle de infecções hospitalares, o meio mais frequente para que haja contaminação e disseminação destas infecções continuam sendo as mãos dos profissionais $^{18}$.

As normativas oficiais brasileiras impõem grande responsabilidade ao enfermeiro no que tange às ações de prevenção das IRAS. A Lei do Exercício 
Profissional de Enfermagem atribui ao enfermeiro a responsabilidade de atuar em conjunto com a equipe de saúde para "prevenção e controle sistemático da infecção hospitalar". A Portaria n².616/1998 estabelece diretrizes e normas para a prevenção e o controle das IRAS e indica a participação preferencialmente de um enfermeiro entre os membros executores dessas diretrizes. O Código de Defesa do Consumidor (CDC) estabelece como dever do Estado garantir a saúde por meio de políticas econômicas e sociais que visem reduzir o risco de doenças e agravos. Diante disto, a participação do enfermeiro no processo assistencial e político é relevante. Ademais, segundo o Código Civil Brasileiro, aquele que por ação ou omissão voluntária, negligência ou imprudência, violar direito e causar dano à outra pessoa, mesmo que moral comete ato ilícito ${ }^{19}$.

Além das responsabilidades diretas sobre suas ações, compete ao enfermeiro coordenar as ações dos demais profissionais da equipe. O desconhecimento das legislações não exime o enfermeiro de suas responsabilidades. $O$ conhecimento dos direitos e deveres éticos e legais é necessário para o adequado cumprimento das obrigações profissionais. Do ponto de vista ético, uma das principais responsabilidades do profissional de enfermagem é assegurar ao paciente, família e comunidade uma assistência livre de danos por imperícia, imprudência ou negligência, bem como protegê-los dessas situações. A atuação do enfermeiro na prevenção de IRAS torna-se complexa sob o ponto de vista ético e legal devido à escassez de recursos humanos, falta de financiamento específico para IRAS, dimensão territorial brasileira com locais de difícil acesso, grande número de instituições e muitos hospitais pequenos com dificuldades para instituir um programa de prevenção de IRAS, oferta assistencial heterogênea e insuficiência de leitos de terapia intensiva ${ }^{19}$.

\section{LAVAGEM DAS MÃOS: MEDIDA EFICAZ CONTRAS AS IRAS}

A interação higienização das mãos $(\mathrm{HM})$ e transmissão de doenças é reconhecida desde o século XIX, proposta primeiramente por Smmelweis, médico que instituiu obrigatoriedade à $\mathrm{HM}$ entre $\mathrm{o}$ atendimento de cada paciente, tendo como efeito a redução da mortalidade. A partir desse momento ficou evidenciado 
cientificamente que a lavagem das mãos poderia evitar a transmissão de patógenos, reduzindo assim os índices de infecções relacionadas à assistência ${ }^{6}$.

As infecções hospitalares ocorrem por diversos motivos e existem muitos mecanismos que favorecem seu aparecimento. Sabe-se que a contaminação através das mãos dos profissionais de saúde representa uma das formas mais importantes de transmissão das infecções em instituições de saúde, principalmente com Staphylococcus aureus e bacilos Gram negativos entéricos ${ }^{20}$.

A higiene das mãos é a medida individual mais simples e menos dispendiosa para prevenir a propagação das infecções relacionadas à assistência à saúde. Recentemente, o termo lavagem das mãos foi substituído por higienização das mãos devido a maior abrangência deste procedimento. $O$ termo engloba a higienização simples, a higienização anti-séptica, a fricção anti-séptica e a anti-sepsia cirúrgica das mãos ${ }^{21}$.

O Ministério da Saúde preconiza que a higienização das mãos se realize quantas vezes forem necessárias para o cuidado com o paciente, sempre que entrar em contato com sítios corporais; entre cada uma das atividades; antes e após entrar em contato com fluidos corporais e secreções; no manuseio de materiais e equipamentos que estiverem em contato com o paciente; no preparo de medicações; antes e após o uso de luvas ${ }^{20}$.

A Enfermagem é a categoria profissional mais envolvida com os cuidados ao paciente, direta ou indiretamente, e, consequentemente, com a profilaxia e controle de infecções relacionadas à assistência, em que a higiene das mãos tem um papel importante 22 .

\section{O ENFERMEIRO ENQUANTO EDUCADOR PERMANENTE}

A educação é algo que faz parte do cotidiano das pessoas, que diariamente aprendem e ensinam nos mais diversos lugares. A educação é algo que invade o cotidiano de trabalho. Para cada grupo de pessoas, existe uma educação diferente, de acordo com o que esse grupo considera importante para fazer parte da formação dos sujeitos. Na enfermagem, as ações são permeadas por espaços educativos que se realizam por meio de diálogos, do conhecimento das coisas, da vida e do 
pensamento. Dessa forma, vislumbram-se espaços potenciais para incentivar transformação e modificar a realidade por meio do conhecimento e da reflexão. Dentre os diversos papéis que os enfermeiros assumem, destaca-se o de educador ${ }^{23}$.

Para que haja mudança de comportamento dentro das instituições de saúde é necessária a escolha de estratégias educacionais aliadas a objetivos claros e que envolvam todos os atores no processo. A educação em saúde objetiva ter valores explícitos diante do problema, aumentar a percepção deste e promover informações e habilidades necessárias para que as decisões sejam corretas $^{18}$.

O comportamento dos profissionais de saúde influencia diretamente na transmissão de $\mathrm{IH}$, uma vez que práticas incorretas funcionam como um veículo de transporte de microrganismos patogênicos ${ }^{24}$. A existência de uma cultura préestabelecida nos serviços de saúde, voltada para o tratamento e a reabilitação, vem dificultando o aparecimento de atividades direcionadas de prevenção de doenças e proteção da saúde ${ }^{9}$.

É no processo de formação que os futuros profissionais poderão adquirir conhecimentos, desenvolver habilidades e consolidar uma atuação com competência na assistência à saúde. A formação superior em Enfermagem deve preparar o profissional para refletir de modo a enfrentar e modificar situações de saúde ou de doença, promovendo a melhoria da qualidade de vida do indivíduo, dos grupos e da coletividade, contribuindo, por extensão, com a transformação da realidade ${ }^{25}$.

Nessa perspectiva, é importante destacar o papel do enfermeiro na prevenção e controle de infecções, pois atua no contato direto com os pacientes, manipula e controla equipamentos, instrumentais e medicações, além de muitos destes profissionais assumirem função de destaque nas Comissões de Controle de Infecções Hospitalares, e de outros espaços no setor da saúde que tratam especificamente desse assunto ${ }^{25}$.

Ressalta-se a relevância da Educação Permanente (EP), como importante estratégia de reestruturação dos serviços, sustentada na análise dos determinantes sociais e econômicos, mas, sobretudo nos valores, atitudes e conceitos profissionais. A EP propõe transformar o profissional em sujeito, colocando-o no centro do processo ensino-aprendizagem, com o intuito de promover um crescimento orientado à 
emancipação, à autonomia e ao compromisso social e político do ser humano e do seu processo de trabalho ${ }^{26}$.

Esta ferramenta é uma alternativa educacional articulada, visando o desenvolvimento de ações voltadas à aprendizagem significativa no e para o processo de trabalho, entendido como eixo para a transformação das práticas, de forma participativa e contextualizada. Atualmente, não basta apenas 'saber' ou 'fazer', é preciso 'saber fazer', imbuído de uma postura profissional permeada de atitudes, valores e um compromisso com a interação entre os sujeitos. Nessa perspectiva, a formação deve ser caracterizada pela autonomia, pela capacidade de aprender constantemente e de relacionar a teoria com a prática ${ }^{27}$.

A formação/educação do trabalhador vai além de ações educativas colocadas pelas instituições, o ideal é que esta formação ajude na transformação dos hábitos e também que o trabalhador reflita sobre sua práxis ${ }^{18}$.

A permanência no hospital, contato com os pacientes, a falta de adesão às medidas de precauções padrão faz com que os profissionais de saúde fiquem sujeitos à colonização por microrganismos tipicamente hospitalares e, frequentemente, multirresistentes, colocando-os na condição de portadores e disseminadores, colaborando para a ocorrência de surtos de infecção ${ }^{28}$.

O controle das IRAS resulta de um esforço conjunto e da adoção de medidas sabidamente eficazes. Esse esforço conjunto depende da vontade de cada profissional envolvido nas ações de saúde, sejam elas preventivas ou curativas. Para a evolução do controle das IRAS, deve-se caminhar para uma conscientização mais profunda a respeito da importância do preparo da equipe hospitalar, que inclui desde conhecimentos mais avançados até a execução de um procedimento mais simples ${ }^{29}$.

Este estudo compreende a educação em saúde como uma possibilidade de cuidado essencial na prática cotidiana, na qual os profissionais de enfermagem podem expandir de forma significativa a sua parcela de contribuição no cuidado aos usuários, às famílias e à comunidade. Diante disso, faz-se necessário que os profissionais de enfermagem, para atuarem com a equipe de saúde e os usuários, estejam constantemente se atualizando em relação à temática e, assim, consigam manter o foco no conhecimento científico, mantendo postura ética e crítica na assistência ao usuário ${ }^{30}$. 


\section{CONSIDERAÇÕES FINAIS}

A partir desta pesquisa, constata-se que a Higienização das Mãos (HM) representa o mais simples e uma das mais importantes medidas de prevenção e controle das IRAS, uma vez que a transmissão microbiana pode ter as mãos como o principal veículo responsável pelas infecções. Na busca do entendimento da relação entre conhecimento e prática, infere-se que os profissionais de saúde têm consciência dos benefícios da HM, porém ainda existem elevados índices de não adesão, mostrando que existe uma lacuna entre teoria e prática.

Atualmente, com tantos meios para o profissional se atualizar, fica até incompreensível afirmar que a baixa adesão da prática de HM provém da falta de conhecimento. Essa prática pode estar mais incorporada aos hábitos dos profissionais do que com seu conhecimento propriamente dito.

É necessário que as instituições de saúde promovam um programa de educação permanente com atividades de orientação voltadas à equipe multidisciplinar, treinamentos, seminários temáticos e reuniões clínicas com 0 envolvimento dos profissionais e a participação efetiva em campanhas, como a de higienização das mãos, adoção de equipamentos de proteção individual, incentivando e promovendo o reconhecimento e valorização dos profissionais.

Apoiado nesse entendimento, este estudo apresenta como inovação a consideração de que a Educação Permanente em Saúde constitui-se em estratégia que respalda o desenvolvimento de ações de Educação em Saúde e Educação em Serviço, desde que sejam operacionalizadas por metodologias que promovam o empoderamento e a qualificação profissional e nisso entra o papel de educador do enfermeiro.

Esta pesquisa não tem a intenção de ser definitiva, mas deve apontar reflexões sobre educação em saúde e sua relação com o processo de trabalho frente ao controle de IRAS, para servir de apoio à qualidade da assistência em saúde. A educação permanente é uma ferramenta para aprimorar os serviços prestados em saúde e também porque transformam as ações do processo de trabalho, dando margem à possibilidade de se atuar de forma crítico-reflexiva, por parte dos trabalhadores de saúde. 
Contudo, para desenvolver pessoas é necessário muito mais do que apenas fornecer informações visando ampliar seus conhecimentos, habilidades e atitudes para se tornarem mais eficientes naquilo que fazem. É preciso proporcionar a formação básica para que aprendam novas atitudes, soluções, conceitos, modifiquem hábitos e comportamentos e se tornem mais eficazes naquilo que fazem.

Soma-se a tudo isso a expectativa de que, por meio dos achados e discussão deste estudo, se consiga contribuir para uma prática de Enfermagem com qualidade e excelência, mais isenta possível de IRAS e um fortalecimento na trajetória da profissão.

\section{REFERÊNCIAS}

1. Silva $A M B$, Andrade $D$, Wysocki $A D$, Nicolussi AC, Haas VJ, Miranzi MAS. Conhecimento sobre prevenção e controle de infecção relacionada à assistência à saúde: contexto hospitalar. Revista da Rede de Enfermagem do Nordeste, vol. 18, núm. 3, Maio-Junho, 2017, pp. 353-360. Universidade Federal do Ceará. DOI: 10.15253/2175-6783.2017000300010.

2. Silva SED, Alves OS, Silva LLS, Sousa DIA, Bitencourt JS, Sá ACC, Filho RFC, Barros KCC, Araujo JSA. Infecção Hospitalar na produção científica da enfermagem brasileira: Um estudo bibliométrico. Revista Eletrônica Gestão \& Saúde, Vol.03, №. 03, Ano 2012: p.1080-93.

3. Sousa CMM, Moura MEB, Santos AMR, Nunes BMVT, Alves MSCF. Responsabilidade civil dos profissionais de enfermagem nos procedimentos invasivos. Rev Bras Enferm, Brasília 2009 set-out; 62(5): 717-22.

4. Paz MCF, Fortes DIFM, Silva DHG. Análise da infecção hospitalar em um hospital universitário na paraíba no período de 2012 a 2014. REVISTA SAÚDE E CIÊNCIA online, 2015; 4(3): 31-43. 
5. Primo MGB, Ribeiro LCM, Figueiredo LFS, Sirico SCA, Souza MA. Adesão à prática de higienização das mãos por profissionais de saúde de um Hospital Universitário. Rev. Eletr. Enf. 2010; 12(2):266-71.

6. Sousa ECP, Silva FL. Conhecimento e adesão da prática de higienização das mãos dos profissionais da saúde: revisão bibliográfica. Rev. Saúde em Foco. Teresina, v. 3, n. 1, art. 1, p. 84-93, jan./jun. 2016.

7. Souza MT, Silva MD, Carvalho R. Revisão integrativa: o que é e como fazer. Einstein. 2010; 8(1 Pt 1):102-6.

8. Pereira MS, Ribeiro LCM, Mendonça KM, Tipple AFV, Souza ACS, Pallos MAP, Barreto RASS. Grupo de Pesquisa em Enfermagem na Prevenção e Controle de Infecções: 20 anos de contribuições. Rev. Eletr. Enf. [Internet]. 2011 jan/mar;13(1):124-9.

9. Cavalcante EFO, Pereira IRBO, Leite MJVF, Santos AMD, Cavalcante CAA. Implementação dos núcleos de segurança do paciente e as infecções relacionadas à assistência à saúde. Rev Gaúcha Enferm. 2019;40(esp):e20180306. DOI: https://doi.org/10.1590/1983-1447.2019.20180306.

10. Oliveira SLT. Análise Bioética das Ações de Prevenção e Controle das Infecções Hospitalares [Dissertação de Mestrado]. Brasília (DF): Universidade de Brasília; 2010.

11. Souza ES, Belei RA, Carrilho CMD, Matsuo T, Yamada-Ogatta SF, Andrade G, Perugini MRE, Pieri FM, Dessunti EM, Kerbauy G. Mortalidade e riscos associados a infecção relacionada à assistência à saúde. Texto Contexto Enferm, Florianópolis, 2015 Jan-Mar; 24(1): 220-8. DOI: http://dx.doi.org/10.1590/010407072015002940013.

12. Silva PLN, Paiva PA, Cruz PKR, Magalhães FR, Souto SGT, Gonçalves RPF, Oliveira VV. Prevenção de infecções hospitalares em pacientes internados em 
unidade de terapia intensiva. Revista da Universidade Vale do Rio Verde, Três Corações, v. 13, n. 2, p. 649-658, 2015.

13. Lara FLO, Antunes AV, Mendes-Rodrigues C, Felice IO. Custos da antibioticoterapia em pacientes adultos com infecção hospitalar em uma unidade de terapia intensive. Rev Pre Infec e Saúde. 2017;3(4):8-14.

14. Michelin AF, Fonseca MRCC. Perfil epidemiológico das infecções hospitalares na unidade de terapia intensiva de um hospital terciário. Revista Nursing, 2018; 21 (236): 2037-2041.

15. Santos SF, Viana RS, Alcoforado CLGC, Campos CC, Matos SS, Ercole FF. Ações de enfermagem na prevenção de infecções relacionadas ao cateter venoso central: uma revisão integrativa. Rev. SOBECC, São Paulo. out./dez. 2014; 19(4): 219-225.

16. Massaroli A, Martini JG. Perfil dos profissionais do controle de infecções no ambiente Hospitalar. Cienc Cuid Saude 2014 Jul/Set; 13(3):511-518. DOI: 10.4025/cienccuidsaude.v13i3.20764.

17. Cabra FW, Silva MZO. Prevenção e controle de infecções no ambiente hospitalar. S A N A R E, Sobral, V.12, n.1, p. 59-70, jan./jun. - 2013.

18. Andrade ES. A importância da educação em saúde para o controle da infecção hospitalar. J Orofac Invest. 2016;3(1):43-52

19. Lamblet LCR, Padoveze MC. Comissões de Controle de Infecção Hospitalar: perspectiva de ações do Conselho Regional de Enfermagem. Revista Cadernos IberoAmericanos de Direito Sanitário. 2018 jan./mar, 7(1):29-42. http://dx.doi.org/10.17566/ciads.v7i1.426. 
20. Pinto FOP, Baptista MA. Higienização das mãos: hábitos, obstáculos, e a técnica desenvolvida pelos discentes do $6^{\circ}$ ano de medicina e do $4^{\circ}$ ano de enfermagem de um hospital escola. Arq Ciênc Saúde 2010 jul-set; 17(3):117-21.

21. Fernandes AT. Percepções de profissionais de saúde relativas à infecção hospitalar e as práticas de controle de infecção [Dissertação de Mestrado]. São Paulo: Faculdade de Medicina da Universidade de São Paulo; 2008.

22. Giarola LB, Baratieri T, Costa AM, Bedendo J, Marcon SS, Waidman MAP. Infecção hospitalar na perspectiva dos profissionais de enfermagem: um estudo bibliográfico. Cogitare Enferm. 2012 Jan/Mar; 17(1):151-7.

23. Lavich CRP, Terra MG, Arnemann CT, Mello AL, Raddatz M. Educação em saúde e educação permanente: ações que integram o processo educativo da enfermagem. Rev baiana enferm. 2018;32:e24719. DOI: 10.18471/rbe.v32.24719.

24. Silva RE, Silva ACA, Freitas AL, Sá CC, Santos IL, Silva VRV, Ventura A, Denadai AS, Fonseca FM. Conhecimento de Estudantes da Área da Saúde Sobre o Controle e Prevenção de Infecções Hospitalares. R bras ci Saúde 22(2):131-138, 2018. DOI: 10.4034/RBCS.2018.22.02.06.

25. Giroti SKO, Garanhani ML. Infecções relacionadas à assistência à saúde na formação do Enfermeiro. Rev Rene. 2015 jan-fev; 16(1):64-71. DOI: 10.15253/21756783.2015000100009 .

26. Cotta RMM, Costa GD, Mendonça ET. Portfólio reflexivo: uma proposta de ensino e aprendizagem orientada por competências. Cien Saude Colet. v. 18, n. 6, p. 18471856, 2013.

27. Amaro MOF, Mendonça ET, Carvalho CA, Nakada KN, Siman AG, Ferreira NCS. Concepções e práticas dos enfermeiros sobre educação permanente no ambiente 
hospitalar. Arq. Cienc. Saúde UNIPAR, Umuarama, v. 22, n. 2, p. 87-94, maio/ago. 2018.

28. Moura JP, Pimenta FC, Hayashida M, Cruz EDA, Canini SRMS, Gir E. A colonização dos profissionais de enfermagem por Staphylococcus aureus. Revista Latino-Am. Enfermagem. Passos (MG), mar/abr 2011.

29. Barros MMA, Pereira ED, Cardoso FN, Silva RA. O enfermeiro na prevenção e controle de infecções relacionadas à assistência à saúde. Universitas: Ciências da Saúde, Brasília, v. 14, n. 1, p. 15-21, jan./jun. 2016. DOI: 10.5102/ucs.v14i1.3411.

30. Dutra GG, Costa MP, Bosenbecker EO, Lima LM, Siqueira HCH, Cecagno D. Controle da infecção hospitalar: função do enfermeiro. J. res.: fundam. care. online 2015. jan./mar. 7(1):2159-2168. DOI: 10.9789/2175-5361.2015.v7i1.2159-2168. 